\title{
Tactile Gloves for Autonomous Grasping with the NASA/DARPA Robonaut
}

\author{
T. B. Martin, R.O. Ambrose \\ Automation, Robotics and Simulation Division \\ NASA Johnson Space Center \\ Houston, TX USA \\ tbmartin,rambrose@ems.jsc.nasa.gov
}

$$
\text { R. Platt, Jr. }
$$

Department of Computer Science

University of Massachusetts

Amherst, Massachusetts 01003

rplatt@cs.umass.edu

\author{
M. A. Diftler \\ Automation and Robotics Dept. \\ Lockheed Martin \\ Houston, Texas 77058 \\ diftler@jsc.nasa.gov \\ M. J.Butzer \\ Human Space Flight Programs \\ Oceaneering Space Systems \\ Houston, Texas 77058 \\ mbutzer@oss.oceaneering.com
}

\begin{abstract}
Tactile data from rugged gloves are providing the foundation for developing autonomous grasping skills for the NASA/DARPA Robonaut, a dexterous humanoid robot. These custom gloves compliment the human like dexterity available in the Robonaut hands. Multiple versions of the gloves are discussed, showing a progression in using advanced materials and construction techniques to enhance sensitivity and overall sensor coverage. The force data provided by the gloves can be used to improve dexterous, tool and power grasping primitives. Experiments with the latest gloves focus on the use of tools, specifically a power drill used to approximate an astronaut's torque tool.
\end{abstract}

Keywords-tactile glove; robot hand; autonomous grasping; humanoid.

\section{INTRODUCTION}

Dexterous humanoid robots offer great potential for assisting humans with a variety of tasks. By definition, they are designed to perform an ever increasing set of tasks that are currently limited to people. Of specific interest here are tasks that currently require human level dexterity while working in dangerous arenas. To this end, NASA and DARPA are jointly pursing the development of a dexterous humanoid robot, Robonaut, for use in the hazardous environments of low earth orbit (LOE) and planetary exploration.

Researchers have been exploring ways of achieving human level dexterity in a robot for several decades. The 3 fingered Stanford/JPL hand [1] and the four fingered Utah/MIT hand [2] were both ground breaking devices. Recently the more compact DLR hand [3], Barrett hand [4] and Robonaut hand [5] have been used to achieve a variety of dexterous, tool and power grasps.

While teleoperation is a feasible starting point for operating a dexterous robot hand. The efficiencies associated with shared control and automation are very desirable: reduced operator work load, manipulation in the presence of time delay, and reduced contact loads. To achieve "good" automation for grasping with a dexterous hand, the location and force level at grasp contacts are necessary. The human analog for grasping and manipulation tasks provides good data for determining this important contact information between a robotic hand and a grasped object.

Human hands and fingers are sensor rich, which allow us to pick up, stably grasp and manipulate objects and tools. These actions require a full sensor set: at the fingertips, all along the finger segments, and on the palm. While many papers describe a number of sensors and sensor types, current research is still active and maturing [6]. There are a number of different technologies being investigated for a wide variety of purposes. Local geometry sensing is being pursued with embedded strain gages [7], and fluid filled membranes [8]. Force Sensing Resistor technology was explored for the DLR three-finger hand [9]. Piezoelectric films have been used for dynamic measurements [10]. New developments in the area of Micro and Nanotechnology have produced promising sensor skins [11]. There is also the promise of commercially available sensors that can be applied to robotic devices $[12,13]$. The primary challenge is to provide enough sensor information to autonomous robotic grasping algorithm developers, in the form of finger and palm coverage, spatial resolution, sensitivity, wire minimization, and robustness.

Autonomous robotic grasping remains a heavily studied research area after almost a quarter of a century of intense research. The approach taken here is closely related to the work of Polly Pook [14] where the Utah/MIT hand was used to autonomously grasp a spatula and flip an egg. In that work, Pook uses a set of context specific control actions embedded in a finite state machine (FSM) to move the system to a desired goal state.

Another related body of work takes a control-based approach to grasping. In this approach, grasp controllers do not assume a specific geometry or pose for the object $[15,16]$. Instead, finger contact information is used to determine a local gradient on a grasp error function. Grasp controllers are 
characterized by a funneling effect on the state of the grasp where the system moves from a large range of initial configurations to a smaller region of more refined grasp states[17]. One or more grasp controllers can be executed concurrently or sequentially to funnel the state of the system into a small neighborhood of good grasp configurations. Recent work has shown the effectiveness of this approach on a simulation of the Robonaut hand [17].

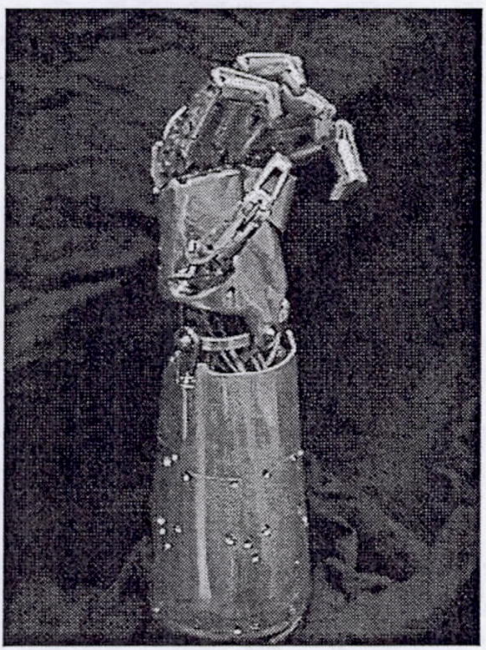

Figure 1. Robonaut hand without glove

\section{ROBONAUT HAND}

The Robonaut Hand [5] has a total of fourteen degrees of freedom. It consists of a forearm which houses the motors and drive electronics, a two degree of freedom wrist, and a five finger, twelve degree of freedom hand (figure 1). The forearm, which measures four inches in diameter at its base and is approximately eight inches long, houses all fourteen motors, 12 separate circuit boards, and all of the wiring for the hand.

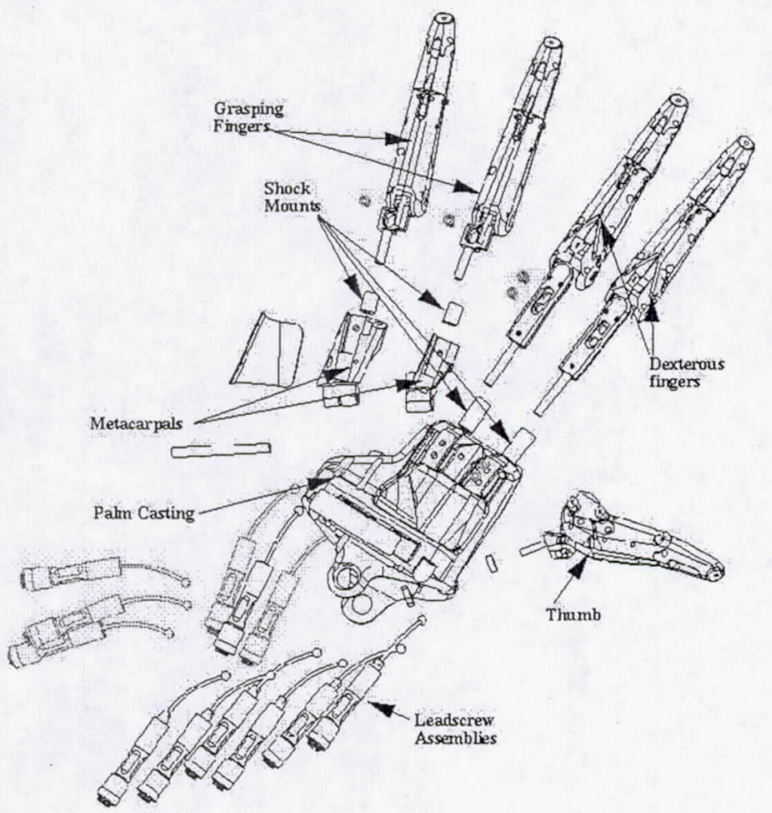

Figure 2. Robonaut hand parts
The hand itself is broken down into two sections (figure 2): a dexterous work set which is used for manipulation, and a grasping set which allows the hand to maintain a stable grasp while manipulating or actuating a given object. This is an essential feature for tool use [18]. The dexterous set consists of two three degree of freedom fingers (pointer and index) and a three degree of freedom opposable thumb. The grasping set consists of two, one degree of freedom fingers (ring and pinkie) and a palm degree of freedom. All of the fingers are shock mounted into the palm.

Under teleoperation control, many tools and objects have been manipulated using the Robonaut hand [19]. In these tests, the operator relied exclusively on visual feedback to obtain and maintain a good grasp. The hand control system limited the force applied by the fingers to prevent damage to any object. Initial automation tests with the Robonaut hand used machine vision [20] to acquire the object, and an arm force sensor to verify the grasp in a very rudimentary fashion. To achieve better grasps, a series of instrumented gloves that contain force sensors has been developed.

\section{FORCE SENSORS}

A large number of candidate sensor types have been evaluated for inclusion into a tactile glove for Robonaut. The types ranged from piezoelectric films to capacitive sensors to carbon resistive films. Many of these initial experiments were carried out on alternative dexterous test beds available within the Automation, Robotics and Simulation Division, at the Johnson Space Center.

The initial sensor suite for the Robonaut glove consisted of Force Sensing Resistor (FSR) technology from Interlink Electronics, Inc.[21]. The FSR sensors have wide appeal, from the standpoint of low cost, good sensitivity, low noise and simple electronics. One drawback is the relatively stiff backing on the sensors, which restricts them from conforming to the curved shape of the Robonaut fingers and palm. Utilizing the smaller off-the-shelf size (approx. 0.25" diameter) allowed for fitting a single FSR sensor onto the smaller finger segments of Robonaut but limited the sensed area. The FSR sensors provided a good capability when integrated into the first generation of Robonaut glove [22]. This initial glove was successfully utilized by visiting researchers conducting grasping algorithm development [17]

Development of new hand/glove sensors is an on-going sub-project for Robonaut. In an attempt to increase the sensor coverage and sensitivity, a new technology was incorporated into the latest generation of Robonaut glove. The basis for the new sensors is a material known as Quantum Tunneling Composite (QTC) developed by Peratech, Ltd., in Durham, England $[23,24]$. The outward effect of the QTC is a change in resistance due to applied pressure. The QTC can be manufactured in sheets of high to low durometer rubber and in various thicknesses. The sheets of QTC are conformal to simple curves and can be cut to fit the irregular shapes of the palm and fingers. The QTC has a very wide dynamic range, from $\sim 10$ MOhms to $\sim 1 \mathrm{Ohm}$, with the application of forces from a fraction of a Newton to 10 Newtons (figure 3). 


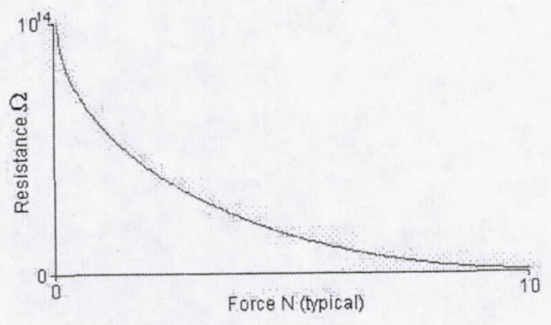

Figure 3. QTC response

The new sensor based on the QTC material is thin, simple and can be adapted to just about any shape. During initial development on a Utah/MIT hand, the QTC-based sensors underwent a number of modifications to increase sensitivity, sensed area, as well as robustness. Many of these modifications were then incorporated into the design of the Robonaut glove.

\section{Robonaut Glove}

Two generations of tactile Robonaut gloves are currently in use with a third version in development. The first version [22] used the Interlink sensors exclusively and was limited to 19 relatively discrete contact points. The second version increases the number of sensors to 33 (figure 4). The large numbers of sensors across the palm are used for both tool and power grasps.

In addition to providing good tactile data, the glove is rugged and designed to protect the sensors, provide excellent gripping surfaces and take the abuse associated with doing a wide range of space based and planetary tasks. Both gloves have incorporated the basic construction of an outer glove with a sensor layer. This allows for assembly of the sensors and wiring independent of most of the sewing and to enhance repair or upgrade of the two layers. The outer glove is a hybrid of an abrasion resistant grip fabric and a flexible lycra blend. The grip fabric is used mostly on the grasping surfaces of the hand where a non-slip robust surface is crucial. The lycra fabric provides good flexibility in the joint areas and creates tension where needed to help position and shape the glove. The sensor layer includes the sensors, force concentrators, wiring, and supporting fabrics. The supporting fabrics include a backing or slip layer next to the finger and adhesive backed fabric to help secure the sensor.

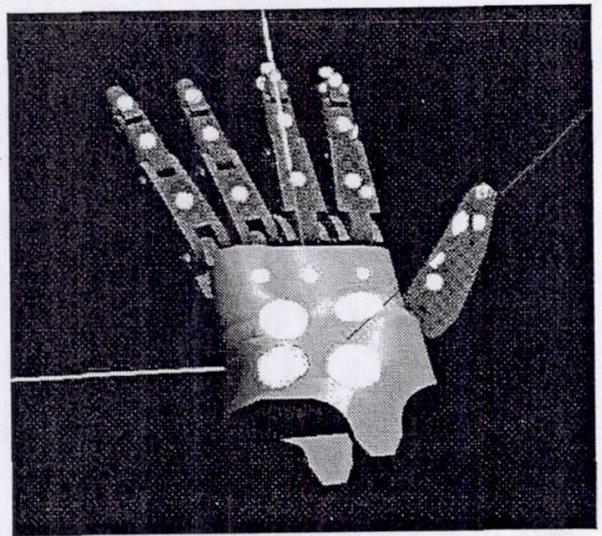

Figure 4. Distribution of sensors

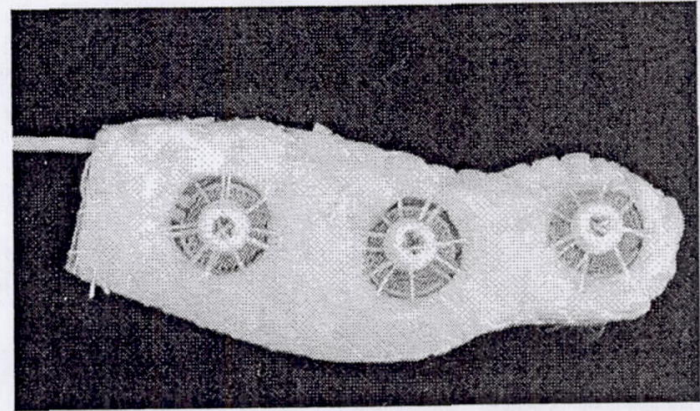

Figure 5. Beads positioned on top of photos inside of upper Palm sensor pockets.

\section{A. Sensor assemblies}

During initial glove development, it was found that the glove layers greatly reduced sensor sensitivity. For the first generation glove, small plastic beads that act as force concentrators were incorporated into the sensor layout to improve sensitivity. In the second generation glove, a variety of force concentrators were explored for both the Interlink and QTC sensor assemblies. In an attempt to reduce construction time and improve flexibility, plus prevent damage to the new sensor types, a medium density rubber with molded hemispherical protrusions was developed and evaluated. The first attempt incorporated protrusions projecting through both the top and bottom of the rubber layer. This provided adequate sensitivity, but made the sensor layout, and subsequently the glove, too bulky. Single sided rubber protrusions were attempted first with the protrusions pointing away from the sensor. This arrangement was discarded due to poor sensitivity. The final attempt to utilize a rubber force concentrator layer pointed the protrusions toward the sensor elements, this improved the sensitivity, but was ultimately discarded for both lack of sensitivity and assembly difficulty. In the end, a combination of durable plastic beads proved to be the easiest solution for both sensor types. The Interlink sensors each have a single bead (figure 5) sized to match the sensor, lashed over the top and secured to the foam layers. The QTC assemblies were somewhat more complex, with a piece of fabric beaded to match the sensor layout on each finger and sewn around the sensor to the backing layer. On both sensors, the bead provides a point load without sharp or rough edges to damage the sensor or the surrounding fabric. The point load creates a high pressure for very light contact forces and yields excellent sensitivity for the assembly.

\section{B. Glove assembly}

When integrating the two glove layers, there must be a careful balance of several factors. Since the outer layer as a whole is flexible and the sensor layer is generally not, sensors and particularly wiring must be supported and located to reduce the risk of damage due to bending, flexing, and stretching. Reduction of bulk is also critical to the overall fit and performance of the glove because it determines the amount of 
work required to open and close the glove with the hand and it can hinder the range of motion of the hand. The extra material required to support and anchor the sensors is carefully located and minimized wherever possible. The most challenging aspect of integration is overcoming the tendency of the glove to exert undesirable forces on the hand.

As the hand and glove change positions, any given area that is loose on an open finger may tighten on a closed finger or vice versa. Forces can also build up within the glove layers as the fabrics bunch or fold when the hand closes. These tendencies are major design drivers in the positioning and mounting of the sensors, because it is desirable to only measure force exerted on or by the hand, not the forces created by the moving glove. This is a key reason to locate sensors between joints of the hand rather than on them. With any sensor type, the correct amount and distribution of slack between the outer layer and the sensor layer was critical. Too much slack added to the problem of bunching or folding and too little could create a tension on the sensor through the force concentrator. Distribution was accomplished by tying the glove layers together in key areas. This approach is not adequate on the Interlink sensors and an additional foam and stiffener layer was required. Foam with a cutout was used to create a recess for the sensor to shield it from the tension and motion of the outer fabric (figure 6). A fabric stiffener was added behind the sensor to protect against folding and bunching along the length of the finger. The QTC assembly proved less problematic and positioning and slack distribution was adequate.

In progressing from the first to second generation glove, techniques were developed that resulted in both thinner and fewer material layers; thereby, resulting in a much slimmer, more sensitive, and more flexible glove.

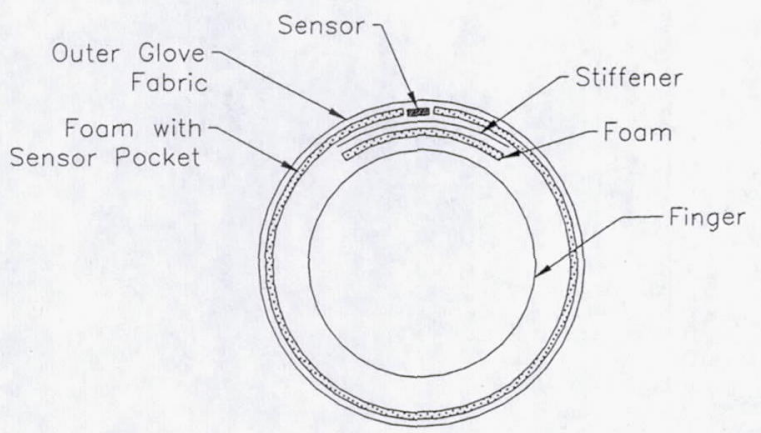

Figure 6. Interlink sensor packaging (cross-section) not-to-scale

\section{Data acquisition and processing}

The current sensor electronics are quite simple and consist only of a set of voltage divider circuits for each sensed area. As the current glove incorporates both FSR and QTC based sensors, the simple circuitry can be utilized for both sensor types, reducing complexity. The choice of the balance resister in the voltage divider network selects the resistance (force) range of interest. The output from the voltage divider is then sent through an $\mathrm{A} / \mathrm{D}$ converter, scaled and made available to display and autonomous grasp routines.

\section{AUTONOMOUS GRASPING}

The first version of the Robonaut glove was used to develop a reflexive grasp and a handrail grasp [24]. The grasping research described here was motivated by a specific task objective. Robonaut was challenged to autonomously grasp a drill in a holster so that the index finger was able to actuate the trigger. The drill and holster were located at an unknown location within some broad region of workspace. After grasping the drill, Robonaut was subsequently required to lift the drill from the holster and engage a bolt elsewhere in the workspace.

In the current work, grasps are synthesized by sequencing a set of context-specific control primitives. The current Robonaut glove does not allow for the calculation of the grasp error function used in [17]. Nevertheless, key control primitives are identified and specially tailored for grasping the drill that exhibit the desired funneling behavior. These primitives move the system through a sequence of grasp states that ultimately result in good drill grasps.

\section{A. Grasp Primitives}

Four grasp primitives make up the drill grasp policy. There is a primitive for moving the hand around the drill handle, another for putting the thumb on the back of the drill handle, another for putting the index finger on the trigger, and finally one for putting the rest of the palm against the handle and closing the fingers.

The first primitive visually servos the hand into a configuration such that the drill handle is between the thumb and index finger. The vision system continually sends updates on the position of the drill handle. The control primitive is preprogrammed with the fixed offset between the handle position and the desired goal position of the hand. This offset is added to the visually determined position of the handle, and the hand moves until it reaches this location.

By observing the kind of grasps used by teleoperators when grasping the drill, it was determined that many good drill grasps placed the base of the inner thumb against the outside of the drill handle. This inspired a grasp primitive that moved the hand until a positive force was registered on the tactile sensor at the base of the inner thumb. This grasp primitive first closes the hand on the drill and observes the pattern of glove tactile sensors registering force. This information is used to generate a motion trajectory that moves the sensor at the base of the inner thumb closer to the drill. The primitive repeats this procedure until the base inner thumb sensor made contact.

One of the main requirements for the drill grasp is that the index finger be able to actuate the trigger. Therefore, the next grasp primitive moves the hand up and down along the axis of the handle until the index finger opposes the trigger. The primitive iteratively closes the index finger on the handle. If the index finger is unable to close, it is assumed that the hand is too high on the handle and the hand moves down the axis of the handle and the procedure is repeated. If the index finger does close, the hand is assumed to be too low on the handle and the hand moves up. This procedure stops when the index finger moves down off the barrel onto the trigger in a single step. 
At this point in the process, the handle and trigger are positioned between the base of the thumb and index finger. The next primitive rolls the hand about the axis between thumb and index finger. The roll continues until a qualitative change in force is perceived on the glove sensors at the bottom of the hand.

\section{B. Sequencing Grasp Primitives}

A successful drill grasp requires that the grasp primitives execute robustly in a specific sequence. If a primitive fails, the system must recover by recognizing the new state of the system and executing the appropriate primitive. To reach the final grasp configuration, the policy is encoded in a finite state machine (FSM).

At each step of task execution, the state of the system is evaluated. State variables include: whether the drill handle is between the thumb and index finger, whether the glove sensor at the base of the thumb is active, whether the index finger is over the trigger, and whether the lower palm is in contact with the handle.

Each state is associated with a specific control action. When the handle is between thumb and index finger, but the thumb sensor is not in contact, the system must put the thumb sensor in contact. Similarly, if the handle is between thumb and index finger, the thumb sensor contact is on, but the index finger is not over the trigger, then it is time to execute the trigger finding primitive.

\section{EXPERIMENTS - DRILL}

The grasp policy is tested by placing the drill in the holster at a number of different positions and orientations and running the grasp policy. The holster is attached to a sliding piece on a long bar that swung about an axis close to Robonaut's body. This allows placement of the holster at an arbitrary position in a plane parallel to the floor. The holster may also be moved through a small range of orientations. See Figure 7.

On each test, grasp quality was measured after the grasp policy completed. If the grasp policy successfully terminated, Robonaut's hand would attempt to pull the drill back out of the holster and actuate the trigger. The grasp was considered successful only if both these tests were successful.

Figure 7 shows the Robonaut gloved hand after making a successful grasp. The key sensors discussed above appear in color in the hand display that is overlaid on the robot's view. Figure 8 shows Robonaut after removing the drill from the holster, note the successful positioning of the thumb and index finger. This view also emphasizes the compact, form fitting design of the glove.

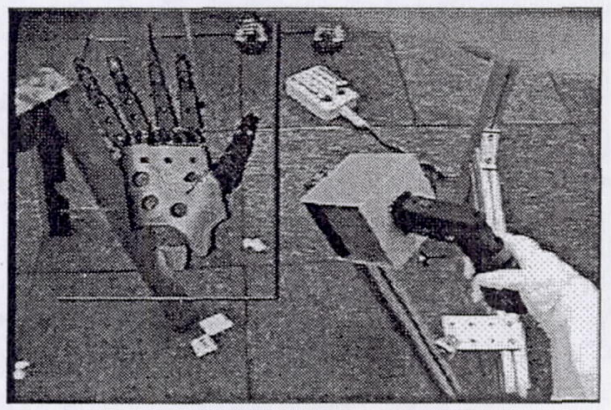

Figure 7. Robonaut gloved hand grasping drill in holster

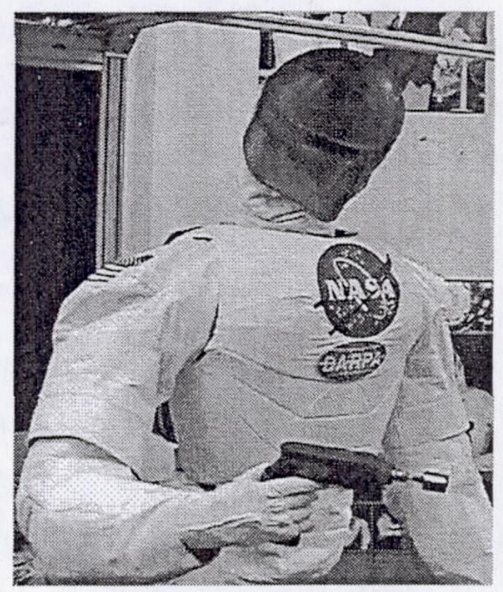

Figure 8. NASA/DARPA Robonaut after acquiring drill

Drill grasps were attempted in approximately 30 different poses. The grasp worked well when the drill was placed within a region of about two feet by one foot. The orientation of the drill varied by about five degrees about one of two axes. Outside of this region, one or another of the primitives failed to accomplish its goal and the grasp policy did not complete. These results indicate that the primitives used in this experiment generalize over only a small set of initial positions and orientations. We suspect that we may be able to generalize over a wider range of initial drill poses by making the primitives themselves more generalizable. Nevertheless, as long as it can be assured that the drill holster will be placed inside this "grasp region", then the grasp policy described here is a useful addition to Robonaut's control repertoire. In particular, this grasp policy can be automatically engaged when the teleoperator moves the hand within a specified proximity to the drill. By taking over and forming the grasp, the grasp policy relieves the teleoperator of some of the more detailed manipulation and allows him to concentrate on higher-level task objectives.

\section{FUTURE WORK}

Robonaut's tactile gloves continue to evolve, providing more contact locations, and greater sensitivity in a slimmer package. The data from these gloves has resulted in a growing set of automated grasping primitives. Future improvements to continue this trend include: 
Improve Sensed Area. While the sensate area of each finger has increased and the palm has nearly full coverage, there are still "dead" areas on the fingers. Further refinement of the sensor pad design and the overall placement of the pads on the finger segments should help alleviate any uncovered areas.

Reduce Wire Count. To further reduce wire count running up the fingers and arm, local signal conditioning and analog to digital conversion is being considered. An added benefit to localized conditioning may be improved spatial resolution. The current compromise is to bus several sensor pads together to reduce wire count, but it also reduces the spatial resolution of the sensor.

\section{ACKNOWLEDGMENT}

NASA and the Mobile Autonomous Robot Software (MARS) program in the DARPA Information Processing Technology Office (IPTO) sponsored this work.

\section{REFERENCES}

[1] Salisbury, J. K., \& Mason, M. T., Robot Hands and the Mechanics of Manipulation. MIT Press, Cambridge, MA, 1985.

[2] Jacobsen, S., et al., Design of the Utah/M.I.T. Dextrous Hand. Proceedings of the IEEE International Conference on Robotics and Automation, San Francisco, CA, 1520-1532, 1986.

[3] Hirzinger, G., Brunner, B., et al., Space Robotics -DLR's Telerobotic Concepts, Lightweight Arms, and Articulate Hands, Autonomous Robots, 14(2/3):127-145, March/May 2003

[4] Townsend, W. T., The BarrettHand Grasper, Industrial Robot: An International Journal, 27(3):181-188, 2000.

[5] Lovchik, C.S., and Diftler, M. A., "The Robonaut Hand: a Dexterous Robot Hand for Space", Proceedings of the IEEE International Conference on Robotics and Automation, Detroit, Michigan, 907-912, 1999.

[6] Lee, M. H., Tactile Sensing: New Directions, New Challenges, International Journal of Robotics Research, Vol. 19, No. 7, pp. 636-643, 2000 .

[7] Provancher, W. R., and Cutkosky, M. R., Sensing Local Geometry for Dexterous Manipulation, Presented at the 2002 International Symposium on Experimental Robotics. July 2002 Sant'Angelo d'Ischia, Italy.

[8] Hristu, D., Ferrier, N., Brockett, R., The Performance of a deformablemembrane tactile sensor: basic results on geometrically-defined tasks, International Conference on Robotics and Automation, 2000
[9] Liu, H., Meusel, P., Hirzinger, G. A., Tactile Sensing System for the DLR Three-Finger Robot Hand, International Symposium on Measurement and Control in Robotics, pp. 91-96, 1995.

[10] Howe, R. D., and Cutkosky, M. R., Dynamic Tactile Sensing: Perception of Fine Surface Features with Stress Rate Sensing, IEEE Transactions on Robotics and Automation, Vol. 9, No. 2, April 1993, pp. 140-151, 1993.

[11] Engle J., Chen J., and Liu C., Development of polyimide flexible tactile sensor skin, Journal of Micromechanics and Microengineering, pp. 359366, 2003.

[12] Son, J. S., Howe, R. D., Tactile Sensing and Stiffness Control with Multifingered Hands, IEEE Robotics and Automation Conference Proceedings, April 1996

[13] Son, J. S., Useful Robots Depend on the Sense of Touch, Pressure Profile Systems, Inc., Application Note, 2002. www.pressureprofile.com.

[14] Pook, P. K. and D. H. Ballard. Sensing qualitative events to control manipulation. Proceedings of the SPIE Sensor Fusion V Conference, November, 1992.

[15] Coelho, Jr. JA, Grupen RA (1997), A Control Basis for Learning Multifingered Grasps, Journal of Robotic Systems, 14(7):545-557. () 1997.

[16] Platt, R., Fagg, A. H., Grupen, R. Nullspace Composition of Control Laws for Grasping, In the Proceedings of the IEEE/RSJ International Conference on Intelligent Robots and Systems (IROS). 2002

[17] Platt, R., Fagg, A. H., Grupen, R. Extending Fingertip Grasping to Whole Body Grasping, Proceedings of the 2003 IEEE Conference on Robotics and Automation (ICRA), Taipei, Taiwan, May 2003

[18] Jau, B., Dexterous Tele-manipulation with Four Fingered Hand System. Proceedings of the IEEE International Conference on Robotics and Automation,. Nagoya, Japan, 338-343, 1995.

[19] Ambrose, R., Culbert, C., Rehnmark, F., An Experimental Investigation of Dexterous Robots Using EVA Tools and Interfaces, AIAA Space 2001, Albuquerque NM, 2001.

[20] Bluethmann, W., Huber, E., et al., A Robot Designed to Work with Humans in Space, Autonomous Robots, 14(2/3):179-197, March/May 2003.

[21] Interlink Electronics, Inc. Force Sensing Resister, Data Sheet, 2002.

[22] Diftler, M. A., Platt Jr, R., Culbert C. J., Ambrose, R. O., Bluethmann, W. J. "Evolution of the NASA/DARPA Robonaut control system", Proceedings of the IEEE International Conference on Robotics and Automation, Taipei, Taiwan, 2543-2548, 2003

[23] Lussey, D.,U.S. Patent No. 6,291,586, 2001, www.peratech.com.

[24] Lussey, D., King, B., Lussey, C., U. S. Patent No. 6,495,069, 2002, www.peratech.com. 